\title{
Low-frequency noises in GaAs MESFET's currents associated with substrate conductivity and channel-substrate junction
}

\author{
DING Yong* \& YAN XiaoLang \\ Institute of VLSI Design, Zhejiang University, Hangzhou 310027, China
}

Received January 24, 2010; accepted April 21, 2010

\begin{abstract}
Low-frequency noises in GaAs MESFET are usually observed when investigating the drain current and substrate leakage current under sidegate bias conditions. Experimental results show that the magnitude of low-frequency noises is in a direct dependency upon the sidegate bias and the noises in drain current will disappear if sidegate bias increases more negatively beyond a certain voltage. A mechanism associated with the substrate conductivity and the channel-substrate junction modulated by sidegate bias is proposed to explain the fluctuation of low-frequency noises.
\end{abstract}

MESFET, low-frequency noise, substrate conductivity, channel-substrate junction

Citation: Ding Y, Yan X L. Low-frequency noises in GaAs MESFET's currents associated with substrate conductivity and channel-substrate junction. Chinese Sci Bull, 2011, 56: 1267-1271, doi: 10.1007/s11434-010-4148-6

MESFET is considered as one of the important devices for the applications of high power, high frequency and high efficiency microwave electric circuit [1,2]. However, as the demand for higher speeds and increased functionality in circuits, low-frequency noises (LFN) in currents, such as thermal noise, $1 / f$ noise, generation-recombination noise, diffusion noise and shot noise, in various GaAs FET structures have been reported [3-10]. The main characteristics of these noises are: (1) LFN strongly depends upon the substrate materials. (2) The frequency of LFN is in the range of about $10^{-2}$ to $10^{2} \mathrm{~Hz}$. (3) LFN is dependent on the fabrication procedure and bias condition. (4) LFN is sensitive to illumination. Recently LFN has received more attention due to its effect on the performance of low-noise microwave integrated circuits (MMICs).

Although it is generally accepted that LFN results from the field-enhanced capture of electrons by deep traps, the exact mechanism is not fully understood. Miller and Bujatti attributed LFN directly to the oscillations in leakage currents in the semi-insulating (SI) GaAs substrate [5]. While Wager pointed out that LFN in channel current could not be

*Corresponding author (email: dingy@vlsi.zju.edu.cn) simply attributed to the oscillations in leakage current of the substrate, but related to the peculiarities of channel-substrate junction [9].

In this paper, LFN in MESFET's currents is investigated under sidegating conditions, and the mechanism is identified whereby the current fluctuation is directly related to the sidegate bias. Unlike Birbas's explanation in terms of an unreal negative capacitance of the depletion region in buffer-channel interface [11], we associate LFN with the substrate conductivity and channel-substrate junction modulated by sidegate bias. This study should be of value to elucidate the fundamental nature of noises modulation in GaAs ICs.

\section{Experiments and results}

The test pattern in this study is shown in Figure 1(a). The device structure and schematic used for electrical measurements are shown in Figure 1(b). FETs are fabricated on undoped liquid-encapsulated Czochralski (LEC) SI GaAs substrate by selective ion implantation. The test device comprises a $10 \mu \mathrm{m} \times 10 \mu \mathrm{m}$ sidegate (SG) and a FET with a 


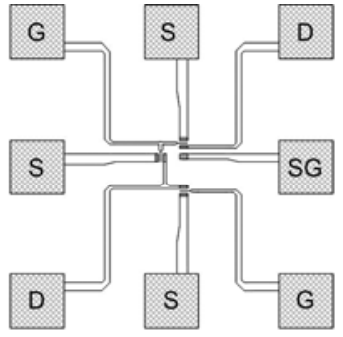

(a)

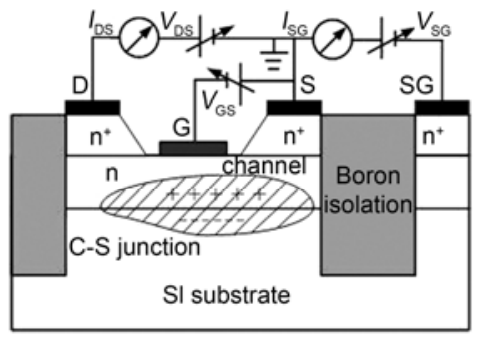

(b)
Figure 1 The test pattern and device structure for investigating LFN. (a) The test pattern used in LFN investigation; (b) the device structure and schematic used for electrical measurements.

$10 \mu \mathrm{m} \times 2 \mu \mathrm{m}$ gate $(\mathrm{G})$. The distance between $\mathrm{SG}$ and the FET is $20 \mu \mathrm{m}$. Silicon ions are implanted at the energy of 60 $\mathrm{keV}$ with a dose of $6 \times 10^{12} \mathrm{~cm}^{-2}$ for $\mathrm{n}$ layer, and at $30 \mathrm{keV}$ with a dose of $1.5 \times 10^{13} \mathrm{~cm}^{-2}$ for $\mathrm{n}^{+}$region. Electrical isolation is achieved by Boron ions implantation at $80 \mathrm{keV}$ with a dose of $8 \times 10^{12} \mathrm{~cm}^{-2}$. Short annealing is carried out at $940^{\circ} \mathrm{C}$ with $10 \mathrm{~s}$. The gate metals used are $\mathrm{Ti} / \mathrm{Pt} / \mathrm{Au}$, and metals for Ohmic contacts are $\mathrm{Au} / \mathrm{Ge} / \mathrm{Ni} / \mathrm{Au}$. The fringe portions of all these contacts and probe pads are in contact with the substrate.

Measurements are made at room temperature with a HP4145B semiconductor parameter analyzer and a Karlsuss testing instrument. The "Integ time" of HP4145B is set to "short", and the "delay time" is set to $1 \mathrm{~s}$. In the experiments, the substrate is kept floating; the source of FET is grounded; the gate bias $\left(V_{\mathrm{GS}}\right)$ of FET varies from $0 \mathrm{~V}$ to -3 $\mathrm{V}$, and the sidegate bias $\left(V_{\mathrm{SG}}\right)$ varies negatively from $0 \mathrm{~V}$ to $-20 \mathrm{~V}$ with a step of $30 \mathrm{mV}$. To investigate LFN in the currents of MESFET, drain current $\left(I_{\mathrm{DS}}\right)$ and substrate leakage current $\left(I_{\mathrm{SG}}\right)$ are recorded.

In the experiments, $I_{\mathrm{DS}}-V_{\mathrm{SG}}$ exhibits a typical sidegating effect as $V_{\mathrm{SG}}$ increases negatively, as shown in Figure 2(a). Sidegating effect is that $I_{\mathrm{DS}}$ of MESFET will decrease abruptly when sidegate bias increases beyond a critical value $[12,13]$. And this critical value is defined as sidegating threshold voltage $\left(V_{\mathrm{SG}}^{\text {th }}\right)$. The sidegating threshold voltage in Figure 2(a) is about -3.8 V. From Figures 2(b)-(f), remarkable LFN can be observed in channel current with sidegate bias varying negatively. In disagreement with the previous work, interesting phenomena are found that: (1) The magnitude of LFN increases as $V_{\mathrm{SG}}$ increases negatively. (2) The oscillations of LFN get the greatest in degree while $V_{\mathrm{SG}}$ reaches $V_{\mathrm{SG}}^{\text {th }}$. (3) $\mathrm{LFN}$ will disappear when $V_{\mathrm{SG}}$ increases more negatively beyond a certain voltage $\left(V_{\mathrm{C}}\right)$. In short, LFN in the channel current of MESFET has a direct dependency upon the sidegate bias conditions. (4) From Figures 2(b) and (f), we can see that the saturation current with $V_{\mathrm{SG}}=-5 \mathrm{~V}$ is less than that with $V_{\mathrm{SG}}=0 \mathrm{~V}$.

In the experiments, $I_{\mathrm{DS}}-V_{\mathrm{GS}}$ is recorded at different sidegate bias, as shown in Figure 3. LFN can also be observed in $I_{\mathrm{DS}}-V_{\mathrm{GS}}$. It can be founded that the noises will also disappear when $V_{\mathrm{SG}}$ increases beyond a certain voltage.

Since it is well known that the sidegating effect is strongly material dependent, some attempts are made to measure LFN in the substrate leakage current. In the experiments, $I_{\mathrm{SG}}$ is recorded as a function of $V_{\mathrm{SG}}$ which increases negatively from $0 \mathrm{~V}$ to $-20 \mathrm{~V}$. The experimental results are shown in Figure 4, in which $I_{\mathrm{SG}}$ exhibits an apparent LFN. Unfortunately, although remarkable LFN can be observed in leakage current, we can not find the same behavior as the LFN in channel current where the LFN will disappear if $V_{\mathrm{SG}}$ increases beyond a certain voltage.

\section{Discussion}

Based on the experimental results presented above, we propose that the substrate conductivity and channel-substrate junction which are modulated by the sidegating voltage take full responsibility for the LFN in currents of GaAs MESFET.

On the one hand, in the sidegating backward biased regime, the resistivity of SI substrate is a variable with different $V_{\mathrm{SG}}$, illustrated as in Figure 5. In general, the resistance of SI substrate $\left(R_{\text {sub }}\right)$ is typically of $10^{7}-10^{9} \Omega \mathrm{cm}$. As $V_{\mathrm{SG}}$ increases negatively, electron emission manifolds gradually in the SI substrate. Thus, the resistivity of the substrate decreases more or less following a linear proportionality to $V_{\mathrm{SG}}$, as illustrated in Region I. If $V_{\mathrm{SG}}$ becomes more negative than $V_{\mathrm{SG}}^{\text {th }}$, the substrate will be taken in the charge of high electric field whose intensity is approximately $10 \mathrm{kV} / \mathrm{cm}$. The free electrons under the high electric field will gain enough energy to excite the trapped electrons in deep traps exhibiting an avalanche multiplication effect. That is to say, impact ionization will take place. In this state, $R_{\text {sub }}$ reduces dramatically with a super linear behavior, as illustrated in Region II. When $V_{\mathrm{SG}}$ reaches a certain voltage where the deep traps are almost fully ionized, $R_{\text {sub }}$ will resume Omhic characteristics, as illustrated in Region III.

On the other hand, as is well known, channel-substrate junction (C-S junction) plays a significant role in sidegating effect of GaAs MESFETs [9,12-17]. The illustration of the C-S junction can be referred to in Figure 1(b). The space-charge distribution of the C-S junction resembles a p-n junction with depletion region located in doped channel and formed by the charge of ionized donors. Expansion of the depletion region on the substrate side under external electric field is accompanied by electrons captured in deep traps, while contraction of the depletion region is accompanied by electrons emission from deep traps to conduction band.

The equivalent circuit of the test structure used in LFN investigation under sidegate bias conditions is shown in Figure $6 . R_{\mathrm{S}}$ is the surface leakage resistance. $R_{\mathrm{CS}}$ is the resistance of C-S junction. With sidegate bias, because $R_{\text {sub }}$ is 

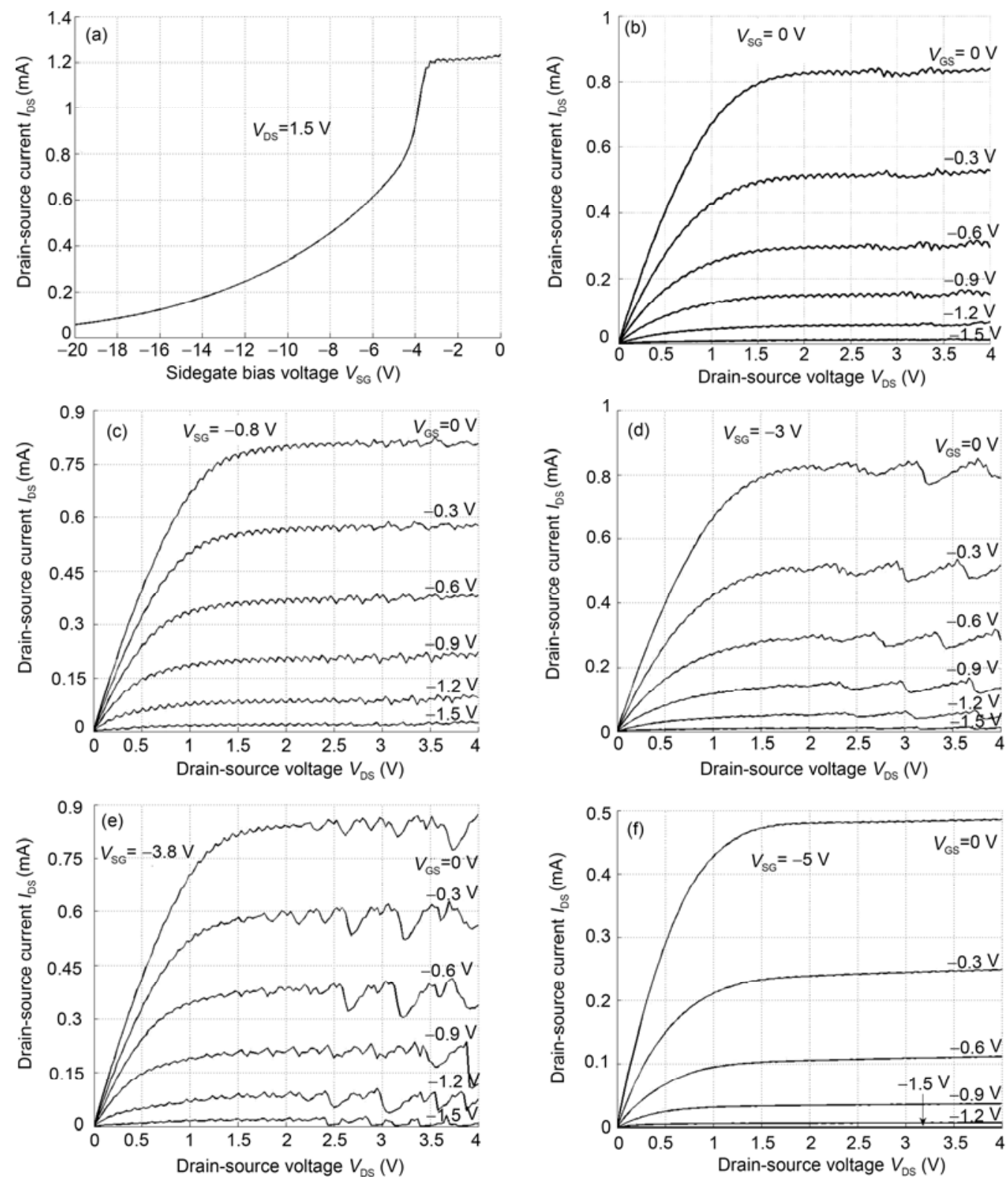

Figure 2 LFN in channel current as sidegate bias increasing negatively. (a) Sidegating effect of GaAs MESFET; (b) LFN in channel current with $V_{\mathrm{SG}}=0 \mathrm{~V}$. (c) LFN in channel current with $V_{\mathrm{SG}}=-0.8 \mathrm{~V}$; (d) $\mathrm{LFN}$ in channel current with $V_{\mathrm{SG}}=-3 \mathrm{~V}$; (e) $\mathrm{LFN}$ in channel current with $V_{\mathrm{SG}}=-3.8 \mathrm{~V}$; (f) LFN in channel current with $V_{\mathrm{SG}}=-5 \mathrm{~V}$.

series connected with $R_{\mathrm{CS}}, V_{\mathrm{SG}}$ partly drops across the substrate (the voltage is denoted as $V_{\text {sub }}$ ) and partly drops across the C-S junction (the voltage is denoted as $V_{\mathrm{CS}}$ ). From the analysis shown above, $R_{\text {sub }}$ varies with $V_{\mathrm{SG}}$, consequently, the voltage drops across the C-S junction changes correspondingly. That is to say, $V_{\mathrm{CS}}$ is modulated by the change of $R_{\text {sub }}$.

The LFN can be analyzed with Khuchua's model of drain current versus $V_{\mathrm{SG}}[12]$,

$$
\begin{aligned}
\frac{\Delta I_{\mathrm{DS}}}{I_{\mathrm{DS} 0}}= & \frac{1}{\sqrt{1+N_{D} / N_{\text {traps }}}} \times \frac{\sqrt{V_{\mathrm{CS}}}}{\sqrt{V_{b}-V_{\mathrm{T} 0}}-\sqrt{V_{b}}} \\
& \times\left(\sqrt{\left.1+\frac{V_{\text {sub }}-V_{\mathrm{SG}}}{V_{\mathrm{CS}}}-1\right),}\right.
\end{aligned}
$$

where $I_{\mathrm{DS} 0}$ and $V_{\mathrm{T} 0}$ are the drain current and the FET threshold voltage at $V_{\text {sub }}=0 \mathrm{~V}$, respectively, $V_{b}$ is the Schottky build-in voltage, $N_{D}$ is shallow donor density, $N_{\text {traps }}$ is the concentration of vacant deep traps.

According to eq. (1), the irregular varieties of $V_{\mathrm{CS}}$ and $V_{\text {sub }}$ give rise to $\Delta I_{\mathrm{DS}}$, thus the fluctuations of $I_{\mathrm{DS}}$ generate. In Region I of Figure 5, as $V_{\mathrm{SG}}$ increases negatively, $R_{\text {sub }}$ decreases more or less following a linear proportionality to $V_{\mathrm{SG}}$. The variety in $R_{\text {sub }}$ will give cause for the increase of $V_{\mathrm{CS}}$ which exerts an influence on the depletion width of $\mathrm{C}-\mathrm{S}$ junction, resulting in the aggravation of oscillations in the depletion width. These oscillations have direct relationship with the fluctuations in channel current and leakage current and they affect the magnitudes of LFN, as shown in 


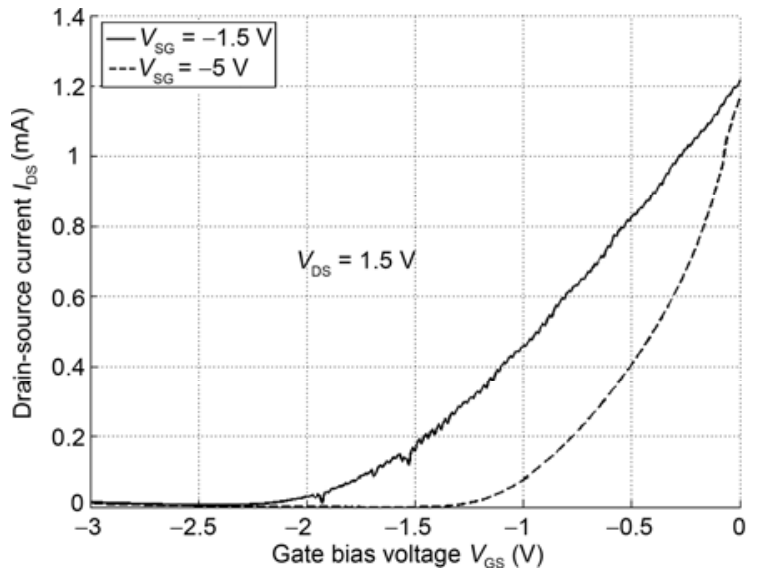

Figure 3 LFN in drain current versus gate bias with different sidegate bias.

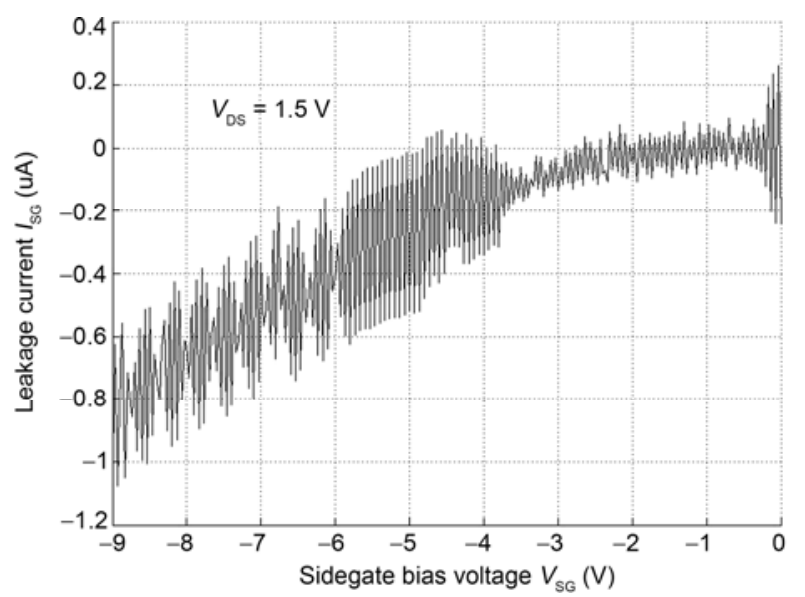

Figure 4 LFN in substrate leakage current as sidegate bias increases negatively.

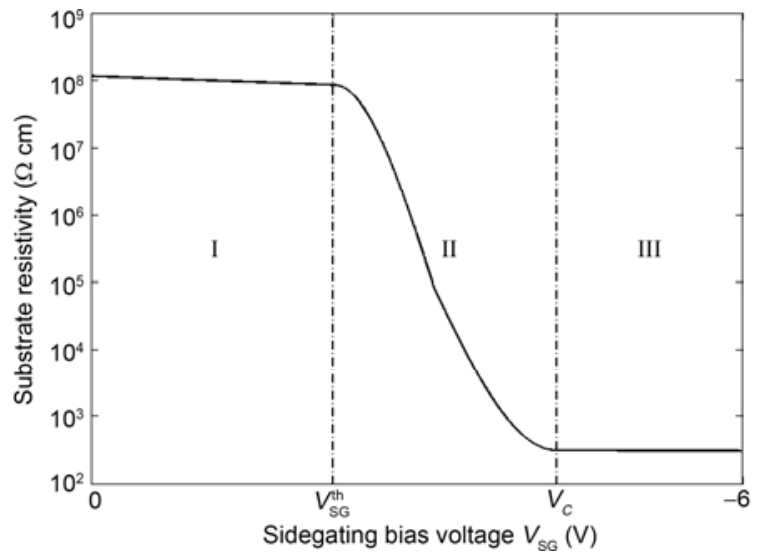

Figure 5 The behavior of substrate resistivity versus backward sidegate bias.

Figure 2(b)-(d) and Figure 4.

In region II, as the superlinear decrease of $R_{\text {sub }}, V_{\text {sub }}$ decreases and $V_{\text {CS }}$ increases dramatically. As a result, an expansion of C-S junction depletion on the channel side mag-

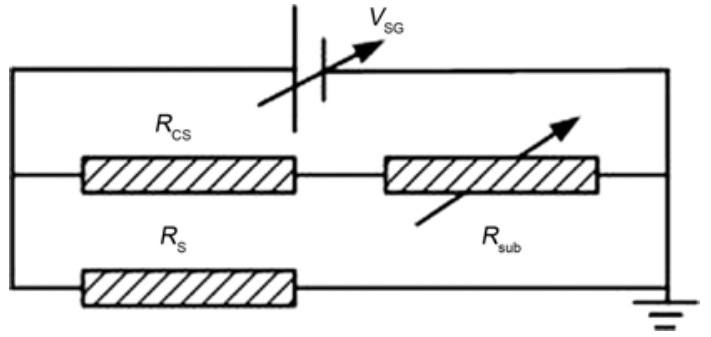

Figure 6 The equivalent circuit of the test structure used in LFN investigation with sidegate bias.

nifies [16]. Meanwhile, there is some hysteresis in the response of the depletion region expansion of C-S junction on the variation of external electric field caused by $V_{\mathrm{SG}}$ [17]. These two factors give rise to larger fluctuations of the depletion region thickness in C-S junction which modulate the effective width of FET channel strongly. In consequence, the fluctuations of LFN in channel current get a great magnitude, as shown in Figure 2(e).

In Region III, $R_{\text {sub }}$ resumes Omhic characteristics and gets stable and low resistance. In this case, the modulation on $V_{\mathrm{CS}}$ caused by $R_{\text {sub }}$ can be negligible, so $V_{\mathrm{SG}}$ mainly drops across the C-S junction. As a result, LFN in current disappears, as shown in Figure 2(f) and Figure 3. Meanwhile, with $V_{\mathrm{SG}}$ becoming more negative, the expansion of C-S junction depletion on the channel side reduces the channel width greatly, the sidegating effect will take place, so saturation channel current declines, as shown in Figure 2(f).

In addition, as shown in Figure 4, although remarkable LFN can be observed in substrate leakage current and it has a direct relation with $V_{\mathrm{SG}}$, it is not found that there is a certain voltage beyond which LFN disappears. A possible cause is that the substrate leakage current recorded in our experiments is only the current from the substrate to the sidegating electrode. In fact, the total leakage current should consist of currents from the FET active region, from the gate pad located on the SI substrate, and from the grounded substrate [5]. In the further research, we will make a thorough investigation on the LFN in leakage current and try to find its relationship with the LFN in drain current.

\section{Conclusions}

LFN in MESFET's currents is investigated under sidegate bias conditions. It is found that the fluctuation of LFN is directly related to the sidegate bias. In disagreement with the earlier work, an interesting phenomenon is observed that LFN will disappear when sidegate bias increases more negatively beyond a certain voltage. It is argued in this paper that LFN in the currents of MESFET associates with the substrate conductivity and channel-substrate junction which are modulated by sidegate bias. 
1 Sun X W, Luo J S, Zhou Z M, et al. A MESFET variable-capacitance analytical model. Chinese Sci Bull, 1997, 42: 374-377

$2 \mathrm{Xu} \mathrm{J} \mathrm{B}$, Zhang $\mathrm{H} \mathrm{Y}$, Wang W X, et al. $200 \mathrm{~nm}$ gate-length GaAs-based MHEMT devices by electron beam lithography. Chinese Sci Bull, 2008, 53: 3585-3589

3 Izpura J I. 1/f electrical noise in planar resistors: The joint effect of a backgating noise and an instrumental disturbance. IEEE Trans Instrum Meas, 2008, 57: 509-517

4 Lin H C, Kim S K, Chang D, et al. Direct-current and radio-frequency characterizations of GaAs metal-insulator-semiconductor field-effect transistors enabled by self-assembled nanodielectrics. Appl Phys Lett, 2007, 91: 092103-1-092103-3

5 Miller D J, Bujatti M. Mechanisms for low-frequency oscillations in GaAs FET's. IEEE Trans Electron Devices, 1987, ED-34: 12391244

6 Wong H. Low-frequency noise study in electron devices: Review and update. Microelectron Reliab, 2003, 43: 585-599

7 Chan Y J, Pavlidis D. Trap studies in GaInP/GaAs and AlGaAs/GaAs HEMT's by means of low-frequency noise and transconductance dispersion characterizations. IEEE Trans Electron Devices, 1994, 41: 637-642

8 Izpura J I. 1/f electrical noise due to space charge regions. J Eur Ceram Soc, 2007, 27: 4011-4015

9 Wager J F, Mecamant A J. GaAs MESFET interface considerations. IEEE Trans Electron Devices, 1987, 34: 1001-1007

10 Ding Y, Lu S L, Zhao F C. Modulation of low-frequency oscillations in GaAs MESFETs' channel current by sidegating bias. Chinese Sci Bull, 2005, 50: 932-935

11 Birbas A N, Brunn B, van Rheenen A D, et al. Low-frequency noise in GaAs MESFETs related to backgating effects. IEE Proc G Circuits, Devices Syst, 1991, 138: 175-178

12 Khuchua N P, Khvedelidze L V, Gorev N B, et al. Determination of deep trap concentration at channel-substrate interface in GaAs MESFET using sidegating measurements. Solid-State Electron, 2002, 46: 1463-1466

13 Zhao F C, Xia G Q, Du L X, et al. Improved analytical model for threshold behavior of sidegating effect in GaAs metal-semiconductor field-effect transistors induced by impact ionization of deep traps. J Appl Phys, 1999, 85: 604-607

14 Horio K, Yanai H, Ikoma T. Numerical simulation of GaAs MESFET's on the semi-insulating substrate compensated by deep traps. IEEE Trans Electron Devices, 1988, 35: 1778-1785

15 Gorev N B, Kodzhespirova I F, Privalov E N, et al. Photocapacitance of selectively doped AlGaAs/GaAs heterostructures containing deep traps. Int J High Speed Electron Syst (IJHSES), 2007, 1: 189-192

16 Li Z M, Mcalister S P, Mcmullan W G, et al. Impact ionization of deep traps in semi-insulating GaAs substrates. J Appl Phys, 1990, 67: 7368-7372

17 Zhao F C, Ding Y, Xia G Q, et al. Hysteresis with nonequilibrium characteristics in sidegating effect of GaAs devices. J Appl Phys, 2000, 87: 1482-1484

Open Access This article is distributed under the terms of the Creative Commons Attribution License which permits any use, distribution, and reproduction in any medium, provided the original author(s) and source are credited. 